\title{
FUENTES Y MÉTODOS PARA EL ESTUDIO DE LOS BANCOS DE RESCATE DE PLATAS EN NUEVA ESPAÑA (1790-1810)
}

\author{
Clara Elena Suárez Argüello*
}

\section{INTRODUCCIÓN}

A l estudiar la manera en que se realizaba el transporte de plata en Nueva España, me fue necesario conocer en qué términos se producía y 1 beneficiaba dicho metal y los actores que participaban en este proceso, para entender más fácilmente cuál era la mecánica que se daba en su traslado; debido a ello fue que descubrí que, a fines del siglo XVIII, se mencionaba en algunos reales mineros la existencia de "bancos de rescate de plata" y que las barras argentíferas eran adquiridas por la Real Hacienda directamente en las cajas reales de los asentamientos mineros.

La añeja problemática del envío de la plata en barras a la Casa de Moneda en la ciudad de México por parte de la minería, que implicaba un tiempo largo por su traslado y retorno en reales efectivos se había modificado, lo que en términos teóricos debía significar la posibilidad de que la minería se financiara de manera inmediata, y no estar sujeta a condiciones onerosas que establecían sus habilitadores al otorgarles préstamos; ello quería decir también que en estas regiones se daría un flujo monetario, al disponer la minería de reales efectivos con que les eran pagadas las barras.

* Centro de Investigaciones y Estudios Superiores en Antropología Social, México, D. F. 


\section{ACERCAMIENTO AL PROBLEMA}

El fenómeno es poco conocido, y la literatura a este respecto es escasa. Una investigación antigua (de 1958) publicada en Sevilla, ${ }^{1}$ y por ello de escasa difusión en México, nos habla de estos bancos. El estudio se aboca a presentar los documentos que a este respecto existen en el Archivo General de Indias (AGI), describiendo el establecimiento de estos bancos en algunas zonas mineras, su operación y los problemas que se enfrentaron con su manejo, pero no se problematiza en torno a las condiciones que tradicionalmente se habían padecido en la minería, ni sabemos tampoco la importancia de sus operaciones, ni qué sucedió con ellos en las décadas previas a la revolución de independencia. Incluso el estudio trata problemáticas ajenas como el doble cobro del señoreaje o el establecimiento del tribunal de minería, que no llevan a explicar el establecimiento y operación de los bancos. ${ }^{2}$

Sin embargo, la publicación nos permite conocer los aspectos contenidos en el material documental, percibiendo la importancia que el establecimiento de estos bancos debe haber tenido en las regiones mineras, dónde se ubicaron y mostrar cuáles fueron ellas. Conociendo la fuente por el estudio, pude remitirme directamente a los documentos alojados en el AGI, analizarlos y considerar que este nuevo planteamiento debió haber alterado en buena medida el manejo tradicional de financiamiento a la minería novohispana. No sólo eso, la tradicional falta de moneda circulante en la economía de Nueva España, que se agudizó a fines del periodo colonial, debió haber sido paliada en estas regiones con la adquisición por parte de las cajas reales de las barras de plata, que eran liquidadas a la minería en moneda contante y sonante.

Un artículo que también refiere a estos bancos es de manufactura más reciente, y en él se realiza una comparación entre los bancos de rescate de platas en México, Perú y Chile, lo que nos permite conocer que el proyecto se aplicó también en diversos reales de minas hispanoamericanos. ${ }^{3}$ El material documental sobre México se basa únicamente en el trabajo citado anteriormente de Pilar Mariscal, que era lo único existente en la fecha de la redacción. La situación es similar para Chile que parece contar con un solo estudio, mientras que para Perú menciona al menos siete investigaciones. El primer punto importante es destacar que las mejoras y cambios realizados en la minería no

${ }^{1}$ Ma. del Pilar Mariscal Romero, Los bancos de rescate de platas, Banco de España/Escuela de Estudios Hispano-Americanos, Sevilla, 1958, 85 pp.

${ }^{2}$ Ibid., pp. 33-47.

${ }^{3}$ Luz María Méndez, "Los bancos de rescate en Hispanoamérica (1747-1832). El proceso histórico y sus fundamentos ideológicos. Estudio comparado para México, Perú y Chile" en Dolores Ávila, Inés Herrera y Rina Ortiz (comps.), Minería colonial latinoamericana. Primera reunión de historiadores de la minería latinoamericana (1). Instituto Nacional de Antropología e Historia, México, 1992, pp. 87-119 (Serie Historia). 
fueron exclusivos para Nueva España, sino que fue la aplicación de una política imperial que abarcó las diversas posesiones hispanoamericanas de España. Está incluida entre las reformas borbónicas, que pretendían impulsar el ejercicio de la minería con el objetivo de aumentar los dividendos de la corona. De esta manera nos enteramos que bajo el nombre de Banco de Rescate del Potosí funcionó en Perú un banco a partir de 1747, que si bien originalmente se encontraba en manos privadas, su administración posterior recayó en la corona. ${ }^{4} \mathrm{En}$ Chile, debido a los importantes yacimientos de plata que se descubrieron entre 1760 y 1780 , se autorizó en 1773 que el rescate de la plata producida se realizara en las cajas reales de Mendoza y San Juan ${ }^{5}$ con dinero de la Real Hacienda, y con esta experiencia se fueron estableciendo diversos bancos de rescate tanto en Perú como en Chile. ${ }^{6}$ En comparación sabemos que los bancos de rescate de plata se instauraron en México más tardíamente, en los inicios de la década de los años noventa.

El documento sobre los bancos de rescate en México que trabajó Pilar Mariscal Romero proviene del Archivo General de Indias. ${ }^{7}$ El legajo permite acercarnos a la problemática del establecimiento de los bancos de rescate, aunque su funcionamiento no queda dilucidado totalmente.

Por él sabemos que en los inicios de la década de los noventa del siglo XVIII se abre un expediente que trata los términos en que éstos se establecieron. La minería de diversos reales inició solicitudes con la Real Hacienda para que sus barras de plata no tuvieran que ser enviadas a la capital, sino que la adquisición se realizara directamente con los reales mineros. La solicitud fue realizada primeramente por los diputados de la minería de Real de Catorce, en donde argumentaban la falta absoluta de numerario para poder realizar sus tareas, y poco después, don Manuel de la Borda (hijo de don José de la Borda) en el real minero de Zacatecas, solicitaba a la Real Hacienda la adquisición de la plata que beneficiaba en su negociación, ya que el financiamiento por parte de los comerciantes rescatadores era leonino y el envío para su cambio a la ciudad de México le implicaba un tiempo excesivo y gastos onerosos, por lo que le era necesario disponer de pesos inmediatos para satisfacer los gastos para que la empresa siguiera en corriente. ${ }^{8}$

Asimismo, en este documento nos enteramos que en uno de los artículos de la reciente Real Ordenanza de Intendentes, emitida en 1786, se contemplaba la problemática que padecía la minería novohispana, ordenando el establecimiento de los bancos de rescate de la plata en las cajas reales de los reales de

${ }^{4}$ Ibid., pp. 95-97.

${ }^{5}$ Ibid., p. 101.

${ }^{6}$ Ibid., véase también de Luz María Méndez, "Bancos de Rescates" en Instituciones y problemas de la mineria en Chile, 1787-1826, Ediciones de la Universidad de Chile, Chile, 1979, pp. 135-146.

${ }^{7}$ Archivo General de Indias (en adelante AGI), Audiencia de México, 2811.

${ }^{8}$ Ibid. 
minas, debiendo adquirir las autoridades hacendarias de la minería local la plata que se produjera, enviando las barras posteriormente a la sede central en la ciudad de México. Consideré importante remitirme directamente a la ordenanza para revisar el artículo 152 que es el referente a los bancos de rescate. ${ }^{9}$ Dicho artículo deja ver los graves problemas que existían en la comercialización de la plata, ya que la minería al necesitar un financiamiento inmediato, entregaba la plata a los mercaderes y rescatadores, que si bien la adquirían a un precio menor que el establecido, también realizaban, al no estar quintada la plata, una serie de negociaciones ilícitas, por lo que la corona se vio necesariamente afectada. Para evitar esta situación, se ordenaba que en las cajas foráneas de los reales mineros se tuviera el suficiente dinero en efectivo para poder efectuar el canje de la plata. El precio que debía pagarse era el que se daba en la Casa de Moneda en la ciudad de México, y dependía de la ley que tuviese el metal.

El citado expediente del AGI, remite también a la problemática que enfrentaba la Real Hacienda para que en las tesorerías foráneas de los reales de minas hubiera el dinero suficiente para poder realizar el canje de la plata. Hay que señalar que la amonedación se realizaba en exclusiva en la Casa de Moneda de la ciudad de México, y el envío de numerario a las cajas reales implicaba el pago del flete del transporte. De la misma manera, el envío de la plata en barras a la Casa de Moneda también tenía un costo, por lo que fue necesario cargar estos gastos a la minería, descontándoles un real por cada marco de plata de cualquier ley. ${ }^{10} \mathrm{Al}$ echar a andar los bancos de rescate de plata, la Real Hacienda envió el caudal que consideró suficiente para que el canje empezara a operar en las cajas reales. De esta manera envió 200000 pesos a la caja real de San Luis Potosí, ya que la minería de Real de Catorce dependía de ella, y a Zacatecas envió otra cantidad igual. Pero seguramente por lo problemático de la operación $\mathrm{y}$ en vías de ahorro, el virrey consideró fundamental que las rentas recaudadas en las regiones mineras por las propias cajas reales, producto de las alcabalas, el tabaco, los naipes, los tributos, las bulas, el papel sellado, la lotería y los fletes que se recibían de los mineros por el azogue, quedaran allí mismo, cubriendo el importe de lo recaudado con barras de plata. ${ }^{11} \mathrm{El}$ expediente nos permite ver cómo, en el transcurso de esta década de los noventa, diversos reales mineros van solicitando el establecimiento de los bancos de rescate, el monto con

${ }^{9}$ Real Ordenanza para el establecimiento e instrucción de Intendentes de Ejército y Provincia en el reino de Nueva España, 1786, Introd. por Ricardo Rees Jones, Universidad Nacional Autónoma de México, México, 1984, pp. 183-184.

${ }^{10} \mathrm{El}$ cobro de este marco por transporte fue específicamente a la minería de Zacatecas; hay que tener presente que los reales de minas se encontraban ubicados a diversas distancias de la ciudad de México, por lo que el costo del flete fue variable.

${ }^{11}$ AGI, Audiencia de México, 2811. 
el que inician sus operaciones, así como el surgimiento de sucesivos problemas que aparecen con el nuevo manejo.

Entre los problemas se encontraba el costo diferencial del transporte según la distancia del real de minas, el valor distinto que se atribuía a las barras de plata, pues uno era el que daba la caja real local y otro el que consideraba la Casa de Moneda, por lo que siempre había una diferencia a favor o en contra, además de incluir en ello el descuento por la afinación de la plata.

De esta manera, el expediente nos permite ver que paulatinamente, a partir de 1791 y hasta 1808, se establecieron bancos de rescate de platas en los siguientes reales de minas: San Luis Potosí, Zacatecas, Pachuca, Rosario, Sombrerete, Zimapán, Durango, Chihuahua, Guanajuato, Bolaños, Guadalajara y Cosalá, mismos que operaron ya sea con el envío de caudales desde la caja matriz de la Real Hacienda o bien sobre las rentas regionales, procurando así que los fondos de rescate permaneciesen siempre íntegros. ${ }^{12}$

\section{UBICACIÓN DEL PROBLEMA}

Profundamente interesada en la problemática de los bancos de rescate de platas, fue fundamental, con base en la información que ya tenía, plantearme una serie de preguntas para poder establecer hipótesis y continuar la investigación.

Si la minería había sido el motor de la economía novohispana durante los tres siglos de colonización, el establecimiento de estos bancos de rescate necesariamente cambiaban el modelo que tradicionalmente había operado. Si bien es cierto que ya desde los inicios del siglo XVIII los grandes banqueros de plata que financiaban a la minería habían perdido importancia, debido a que a partir de 1733 la corona intervino directamente en el manejo de la Casa de Moneda, implicó el retiro de éstos en el proceso de amonedación..$^{13}$ Entre las medidas tomadas se encontró la dotación a la Casa de Moneda de un fondo revolvente de 500000 pesos, lo que permitió a los mineros el canje inmediato de sus barras de plata, aunque desde luego desde la ciudad de México. Sin embargo, la habilitación a las minas continuó siendo fundamental, ya que el envío directo de las barras de plata a la Casa de Moneda implicaba -según la distancia del real de minas a la ciudad de México- el gasto del flete y un tiempo muerto, ya que a la minería le era necesaria una erogación constante de dinero para el pago de rayas de sus operarios y la adquisición de insumos indispensables para

${ }^{12} \mathrm{Ibid}$., 2235, referente al establecimiento del banco de rescate de oro y plata en Guanajuato.

${ }^{13}$ David A. Brading, Mineros y comerciantes en el México borbónico, Fondo de Cultura Económica, México/Madrid/Buenos Aires, 1975, pp. 197-198; Víctor Manuel Soria Murillo, La casa de moneda de México bajo la administración borbónica, Universidad Autónoma Metropolitana, México, 1994, pp. 28-32. 
su funcionamiento. No obstante, en los reales de minas siempre existieron los denominados "rescatadores" que adquirían tanto "el partido" sacado por los operarios mineros como la plata obtenida por sus propietarios, pagándola por supuesto a un precio considerablemente más bajo. Dichos rescatadores, en su mayoría comerciantes de la localidad, se encontraban estrechamente vinculados con los comerciantes de la ciudad de México, que en buena medida les financiaban sus operaciones. ${ }^{14}$ De esta manera, la función de habilitación y avío a la minería por parte del comercio continuaba, ya que los mineros, dada la naturaleza de su trabajo, no disponían de recursos con un flujo constante. A pesar de los grandes beneficios que se otorgaron a la minería en la segunda mitad del siglo XVIII, a raíz de la visita de José de Gálvez, tales como la baja en el precio del azogue y de la pólvora, la supresión del pago de alcabalas para los insumos que les eran necesarios, la creación del Tribunal de Minería que debería apoyarlos con créditos, la necesidad de disposición inmediata de fondos era ineludible, por lo que la habilitación a los mineros y el rescate de la plata en pasta continuó por parte de los mercaderes, aunque muchos de éstos, al incrementarse las ganancias de la minería por la rebaja en los costos de producción, empezaron a invertir sus caudales directamente asociándose con los mineros..$^{15} \mathrm{El}$ establecimiento de fondos de rescate para la plata en los reales de minas por parte de la Real Hacienda, fue el jaque mate para los "rescatadores", ya que el pago que realizaban los bancos de rescate era el mismo que daba la Casa de Moneda, descontando solamente el flete de ida y vuelta a la ciudad de México y los derechos correspondientes, que ya se pagaban tradicionalmente. La Real Hacienda se benefició al disminuir la circulación de la plata sin quintar, y también los mineros al lograr de manera inmediata el canje de su plata.

La pregunta evidente era ¿qué significó este cambio para la minería? Seguramente el que los mineros dispusieran ellos mismos de los reales efectivos debió haber sido una inyección económica para toda la población del real minero y las regiones circundantes. ¿Estos bancos de rescate implicaron un fomento a la producción minera?, ¿se palió la escasez de circulante en estas regiones, fenómeno tan mencionado a fines del periodo colonial?, ${ }^{16}$ ¿la operación de estos bancos permitió el acceso a todos los mineros? Y extrapolando un poco, también me hice una pregunta sobre la autonomía de estas regiones al disponer ya de su propio capital. También consideré cómo una solución aparentemente tan sencilla no había sido llevada a cabo con anterioridad.

${ }^{14}$ Pedro Pérez Herrero, Plata y libranzas. La articulación comercial del México borbónico, El Colegio de México, México, 1988, pp. 114-118.

${ }^{15} \mathrm{Ibid}$., pp. 145-149. Otros fenómenos tales como la promulgación de la libertad de comercio, influyeron también en el establecimiento de estas sociedades.

${ }^{16}$ Ruggiero Romano, Moneda, seudomoneda y circulación monetaria en las economías de México, El Colegio de México, México, 1998. 
Para poder responder a todo ello, había que seguir muchas etapas, algunas de las cuales se han satisfecho, aunque todavía queda un largo camino por recorrer.

\section{ANTECEDENTES DE LOS BANCOS DE RESCATE}

Sabía que la problemática del rescate de plata era un problema añejo, por lo que me di a la tarea de encontrar los antecedentes para profundizar en el tema. Me interesaba saber cómo se había manejado el asunto y cuáles habían sido las respuestas oficiales. Una fuente fundamental para el conocimiento de la minería en la época es la obra de Francisco Xavier de Gamboa; ${ }^{17}$ en otras fuentes consultadas observamos los sucesivos intentos que hubo tanto de autoridades como de particulares para resolver dicha problemática. ${ }^{18}$ Las propuestas, emitidas las primeras desde el siglo XVI por Felipe II en las ordenanzas de minería, continuaron sucesivamente hasta el siglo XVIII, ${ }^{19} \mathrm{y}$ de una $\mathrm{u}$ otra manera contemplaban la existencia de un fondo revolvente en algunas provincias consideradas clave, que permitiría la adquisición de la plata pasta y evitaría tanto la escasez de circulante como las condiciones leoninas que imponían los mercaderes. ${ }^{20} \mathrm{~A}$ lo largo de estos tres siglos se hicieron una serie de propuestas y se trató de paliar la problemática, pero también descubrimos que las soluciones eran obstaculizadas por los propios mercaderes, que no permitían se extinguiera la fuente de sus ganancias. Inclusive el recién creado tribunal de minería, instituido por la iniciativa de Gálvez, trató de ser una solución para el fomento de la minería, mediante los préstamos que otorgaría y que fracasó por la corrupción

${ }^{17}$ Francisco Xavier de Gamboa, Comentarios a las ordenanzas de minas, Miguel Ángel Porrúa, México, 1987 (edición facsimilar de la de 1761); también se revisaron los trabajos de Fausto de Elhuyar, Memoria sobre el influjo de la mineria en Nueva España, Consejo de Recursos Naturales no Renovables, México, 1964 e Indagaciones sobre la amonedación en la Nueva España. Miguel Ángel Porrúa, México, 1979 (reproducción facsimilar de la de 1818).

${ }^{18}$ Elías Trabulse, "Francisco Xavier de Gamboa y sus comentarios a las ordenanzas de minas" en Fco. Xavier de Gamboa, op. cit., pp. 34-40. Asimismo véanse pp. 421-422; Brading, op. cit., pp. 219-231, Pérez Herrero, op. cit., pp. 142-143.

${ }^{19}$ AGI, Estado, 34, núm. 35, fs. 132-157, Memoria de Josef de Gálvez sobre el estado de América: México; Biblioteca Nacional de Madrid, Mss. 10390, Informe que en virtud de real orden de 24 de mayo de este año hizo el Ilmo. Sr. visitador general don José de Gálvez al Excmo. Sr. Virrey don Antonio Ma. Bucareli...; Ibid., Mss. 2929, Instrucción del duque de Linares, Gob. de Nueva España para su sucesor, con descripción geográfica y política de su gobierno e informe de los males que padecía; Biblioteca del Palacio Real de Madrid. Miscelánea de Ayala, II/2823, fs. 96-100v. Proyecto que propone don Domingo Ruberato y Solar de una compañía de minas en las provincias del reino de Nueva España, que con 2000000 de fondos ofrece evidente aumento a los haberes de S. M. e igual interés a sus vasallos....

${ }^{20}$ Para más detalle de todo este proceso veáse Clara Elena Suárez Argüello, "Los bancos de rescate de platas iincremento en la circulación monetaria y ahorro de fletes? Una nueva propuesta a fines del siglo XVII", Relaciones, vol. Xx, núm. 79, verano de 1999, pp. 92-98. 
imperante y la intromisión de la corona que sacaba dinero para su beneficio. El propio Gálvez fue explícito en proponer que en las cajas reales de los centros mineros hubiera los recursos suficientes para la adquisición de las barras de plata. Así lo señala en el informe que le dejó a Bucareli, ${ }^{21}$ y que aparece redactado ya como un artículo en la Ordenanza de intendentes.

\section{LA OPERACIÓN DE LOS BANCOS DE RESCATE}

Si bien conocíamos que los bancos de rescate de plata se habían ido instaurando paulatinamente a partir de la década de los noventa del siglo XVIII en diversos reales de minas, poco sabíamos de cómo habían operado y por cuánto tiempo. La búsqueda de material en el Archivo General de la Nación de México fue fructífera, pues en el ramo de Minería encontramos algunos volúmenes que tratan el tema. ${ }^{22}$ Los diversos expedientes al respecto nos hablan tanto de la instauración de los bancos en diversos reales, como de los problemas que se enfrentaron para su operación. ${ }^{23}$ Por supuesto, como la mayoría de los expedientes históricos proporciona una información fragmentada que hay que completar con las argucias que se le ocurran al investigador. Por supuesto, son documentos oficiales escritos por las propias autoridades, y poco sabemos cuál fue la reacción del gremio de mineros del real o de la población. Parte de estos documentos, obviamente, repiten la información encontrada en el AGI, ya que muchos de ellos se remitían a los funcionarios metropolitanos. Pero la información en muchas ocasiones es muy rica y nos permite avanzar en la investigación. Así, por ejemplo, un informe nos permite saber que los bancos de rescate operaron algunos años a partir de su establecimiento ${ }^{24}$ y nos da un panorama que permite conocer a detalle el número de barras de plata intercambiadas, el valor que se pagó por ellas en la caja real de la localidad y el valor que le dio la Casa de Moneda. ${ }^{25}$

${ }^{21}$ AGI, Estado, 34, núm. 35, fs. 132-157, Memoria de Josef de Gálvez sobre el estado de América: México. Biblioteca Nacional de Madrid, Mss. 10390. Informe que...

${ }^{22}$ Archivo General de la Nación (en adelante AGN), Minería, expedientes de los volúmenes 82, 83 y 84 .

${ }^{23}$ Los expedientes de estos volúmenes tratan del establecimiento de bancos de rescate en Rosario, en Real de Catorce, Sombrerete, Zimapán, etc., que se estudia la posibilidad de aplicar libranzas para los rescates, el propio comandante general de las provincias internas para que se establezcan rescates en Durango y Chihuahua, sobre las diferencias notadas en la plata y oro de rescate, que se asigne compensación a los funcionarios que realizan los rescates, etcétera.

${ }^{24}$ Dado que el establecimiento del banco de rescate en cada real minero fue en fechas diferentes, el informe mencionado incluye al banco instalado en la caja real de San Luis Potosí y abarca de 1791 a 1796; el de Zacatecas y Pachuca abarca estas mismas fechas; Sombrerete de 1792 a 1796, Rosario de 1793 a 1796, y el de Chihuahua sólo abarca 1794 y 1795.

${ }^{25}$ AGN, Minería, vol. 84, exp. 3, fs. 58-60. También en AGI, México, 2811, fs. 8-8v. 
Esta información nos permitió saber la envergadura e importancia de los bancos de rescate. A simple vista, la cantidad inyectada en cada real era suficientemente significativa, pero para conseguir un parámetro más sólido, se decidió compararlo con lo que se acuñaba anualmente en la Casa de Moneda de la ciudad de México. ${ }^{26}$ Y si bien en los primeros años de operación de los bancos de rescate la operación fue poco significativa (1.06\%-7.67\%), de 1794 a 1796 la proporción de dinero manejado en el rescate oscila entre 11.45 y 14.50 por ciento.

Si consideramos en dinero circulante estas proporciones, significaron aproximadamente 3000000 de pesos, que repartidos en seis localidades mineras deben haber significado un buen alivio a la escasez de circulante que se padecía. Sin embargo, consideramos que había que profundizar mucho más en la investigación y que el sondeo en otro tipo de documentos nos permitirían una mejor perspectiva. Por lo pronto, dejamos de lado otros problemas referentes al transporte y los fletes, íntimamente vinculados a los bancos de rescate y las dificultades que encontraron las autoridades en el manejo de los bancos para conocer cuánto tiempo operaron realmente, los montos manejados y el posible impacto que debieron haber tenido en las regiones.

\section{UNA ALTERNATIVA PARA PROFUNDIZAR EN LOS BANCOS DE RESCATE}

A pesar de tener un panorama más o menos amplio de la operación de los bancos de rescate, me sigue intrigando qué tanta importancia habían tenido éstos en la economía novohispana, y el impacto que habían causado entre la población de las diversas regiones.

Por otro lado, trabajar en otro proyecto con documentación sobre Zacatecas me llevó a considerar la posibilidad de estudiar cómo había operado el banco de rescate en este real. La correspondencia sostenida por dos de los administradores de una gran empresa minera, que actuaba precisamente en los años de la operación de los bancos de rescate, me brindó una perspectiva diferente, ya que se trataba de las cartas que escribían informando sobre los avances o problemas que enfrentaba la negociación y describían los sucesos que afectaban la vida de Zacatecas. ${ }^{27}$

Profundizar en el caso de Zacatecas tenía grandes ventajas; además de enriquecer el estudio con los documentos mencionados anteriormente, había una sólida plataforma con investigaciones ya realizadas. El exhaustivo trabajo

\footnotetext{
${ }^{26}$ R. Romano, op. cit., p. 30; Biblioteca Nacional de Madrid, Mss. 13210, f. 412.

${ }^{27}$ Fue por la invitación que me hizo la doctora Brígida von Mentz a colaborar con ella en el proceso de paleografiar la correspondencia y cuentas de la Compañía de Vetagrande, que encontró en la Southern Methodist University, Dallas, y que preparamos para su publicación.
} 
de Richard Garner, que desafortunadamente no fue publicado, ${ }^{28}$ y el trabajo relativamente reciente de Frédérique Langue, ${ }^{29}$ me aportaron un panorama general de las condiciones de vida en Zacatecas a fines del siglo XVIII. Si a ello sumamos el generoso archivo histórico ${ }^{30}$ ubicado en Guadalupe, Zacatecas y la Zacatecas Collection que contiene los documentos de la caja real zacatecana, provenientes de la Clements Library, University of Michigan y que fueron enviados para su resguardo al Instituto Tecnológico de Monterrey, campus Zacatecas, ${ }^{31}$ consideré que tenía elementos más que suficientes para poder redondear la operación del banco de rescate de plata. ${ }^{32}$

Para mi fortuna, en la Zacatecas Collection encontré los registros que mostraban los canjes de barras que la caja real había realizado desde 1791 hasta 1803. Este padrón no solamente me dio los totales de barras canjeados en cada año y su valor en pesos, sino que también me indicó qué individuos realizaron el cambio, las fechas en que lo realizaron, cuántas barras llevaron, de qué peso y el tipo de beneficio usado. Por lo pronto decidí vaciar esta información a una base de datos que incluye la información mencionada. Esta tarea hasta ahora incluye de 1791 a 1794, con un total de 2572 registros, y si bien es un trabajo arduo por los resultados a la fecha, creo que vale la pena.

Además de conocer la envergadura de la operación del banco de rescate, la base de datos nos permite saber quiénes realizaban los canjes de barras de plata, y con ello darnos cuenta si eran los propios mineros los que hacían los cambios o seguían acudiendo los mercaderes. También podemos saber la importancia de las operaciones realizadas, la cantidad de plata que canjeaban y el monto de dinero que por ello conseguían. Pensamos que cuando se termine de vaciar la información de los años faltantes (de 1795 a 1803) podremos saber con mayor precisión quiénes fueron los realmente beneficiados con estas operaciones. También podremos tener un panorama general de la minería zacatecana, al comparar el monto de plata canjeada por el rescate con la producción anual total de su minería, lo que nos permitirá ver el impacto del banco de rescate.

Por lo pronto, hemos podido comparar las cantidades globales que nos proporcionan los propios informes de la Zacatecas Collection ${ }^{33}$ y la producción

\footnotetext{
${ }^{28}$ Richard L. Garner, "Zacatecas, 1750-1821: the Study of a Late Colonial Mexican City", tesis de doctorado, University Microfilms International, Dissertation Information, 1970.

${ }^{29}$ Fréderique Langue, Los señores de Zacatecas. Una aristocracia minera del siglo XVIII novohispano, Fondo de Cultura Económica, México, 1999.

${ }^{30}$ Archivo Histórico del Estado de Zacatecas (AHEZ), Guadalupe, Zacatecas.

${ }^{31}$ Zacatecas Collection, Instituto de Estudios Superiores de Monterrey, campus Zacatecas.

${ }^{32}$ Clara Elena Suárez Argüello, "Los bancos de rescate de platas: ¿una opción alternativa para el financiamiento de la minería? El caso de Zacatecas: 1791-1810" en Guillermina del Valle, Mercaderes, comercio, consulados en Nueva España en el siglo XVIII, Instituto Mora, México, en prensa.

${ }_{33}$ ITESM, Campus Zacatecas, Zacatecas Collection, Treasury Records and Correspondance, Barreaje.
} 
total de plata del real de Zacatecas que nos presenta Garner, ${ }^{34}$ y consideramos que el impacto debió haber sido suficientemente significativo, ya que el porcentaje promedio del rescate en relación con la producción total de plata en Zacatecas fue de $\mathbf{4 5 . 5 7 \%}$. También hay que señalar que nos hemos apoyado para tener un parámetro más de comparación, en los ingresos y egresos que tenía la caja real de Zacatecas: ${ }^{35}$ mientras que los ingresos provenían del cobro de diversos impuestos y del pago del azogue que realizaba la minería zacatecana, los egresos consistían en el pago de sueldos a los diversos funcionarios, el monto destinado a la operación de rescates y lo que se enviaba a la caja matriz de la Real Hacienda en la ciudad de México. De esta manera tenemos que los montos operados por los bancos de rescate en relación con los ingresos de la caja real significaron desde $27.38 \%$ (en 1801 , el año de menor importancia) hasta $82.06 \%$ (en 1802).

Uno de los ejercicios que hemos realizado es detenernos a analizar un año específico, con el objetivo de comparar detenidamente tanto el monto de ingresos como de egresos de la caja real, y los movimientos llevados a cabo por el banco de rescate. El año elegido, fue el de 1793, pues ya se encontraba desglosado totalmente en la base de datos y disponíamos de la información de las operaciones realizadas por la caja real ${ }^{36} \mathrm{El}$ análisis de estos datos nos permite ver la importancia de los movimientos comerciales (alcabalas, tabaco, bebidas alcohólicas, pulperías, etc.), qué tan significativa era la población menos privilegiada (tributos) y la importancia de la producción argentífera, medida en los derechos de ensaye, los derechos de la plata de azogue y fuego, los del oro, y el pago que realizaban los mineros por el azogue que recibían. Asimismo, la comparación de los ingresos totales de la caja real con respecto al movimiento realizado en las operaciones de rescate, nos provee de elementos para ver su importancia, además de que otro parámetro de comparación lo tenemos con los datos de la producción total de plata en el lugar. ${ }^{37}$ El monto de dinero canjeado en este año por el banco de rescate en relación con la producción total de plata del real de Zacatecas fue de $39 \%$, mientras que la erogación realizada en relación con los ingresos totales de la caja real fue de 53\%. Esta información nos permite afirmar, cuando menos para este año, que el rescate de plata realizado en la ciudad de Zacatecas tuvo un impacto real para su economía y la de la región circundante.

${ }^{34}$ Richard Garner, Zacatecas, op. cit., pp. 215-216.

${ }^{35}$ Richard Garner, "Zacatecas. Spanish Treasury Data Bank" en http://mansell.stucen.gatech. edu/rlg7/latamcaja/index.html, 2000, pp. 84-98, basado en Te Paske y Klein. Véase también R. Garner, Zacatecas, op. cit., pp. 107-108.

${ }^{36}$ AGI, Guadalajara, 481, Tesorería de Real Hacienda, Zacatecas, 1793.

${ }^{37}$ R. Garner, Zacatecas, op. cit., pp. 215-216. 
La información que le dimos a la base de datos sobre este mismo año de 1793 , nos permitió profundizar y matizar lo dicho hasta ahora. ${ }^{38} \mathrm{Si}$ bien los canjes de plata fueron realizados durante todo el año, el total de personas que lo realizaron fueron 31 , pero solamente unas cuantas fueron las que cambiaron un número mayor de barras; tres de ellas cambiaron más de 100 barras, otra estuvo muy cerca (94), mientras que las 27 restantes permutaron entre una y 20 barras. El número total de barras entregadas para el canje fue de 690 , por lo que a simple vista nos damos cuenta que una minoría fue la que aprovechó mayormente el rescate. ${ }^{39}$ Las personas restantes realizaron operaciones de mucho menor monto, que fueron desde los 534 pesos hasta los casi 20000 pesos; sólo uno más alcanzó un monto de 31664 pesos.

El paso siguiente fue tratar de saber quiénes eran los individuos que realizaban los cambios de barras de plata, y sustentada tanto en los estudios anteriormente mencionados como con la información obtenida del Archivo Histórico de Zacatecas, fue relativamente fácil ubicar a algunos de estos personajes. Hemos detectado que los que cambiaron más barras de plata eran mineros de profesión o los que trabajaban en alguna hacienda de beneficio de plata. Pertenecían a la elite minera y eran miembros o tenían acciones en las grandes empresas mineras que funcionaban a fines del siglo XVIII, tales como Vetagrande o Quebradilla. El grupo restante estaba conformado en su mayoría por comerciantes, en algunos casos también dedicados a la minería, pero que habilitaban a otros mineros o les adquirían la plata que obtenían. Hay que mencionar también que en esta época era usual tener varios empleos, por lo que no era raro que un individuo además de poseer una pulpería, tuviera intereses en la minería.

Aún queda un largo camino por recorrer: concluir el vaciado de la información de los registros de rescate de platas de 1795 a 1803; buscar en la "Zacatecas Collection" el resto de información que refiera a los años de 1804 en adelante; averiguar lo sucedido a raíz de la guerra de Independencia y tratar de ligar si efectivamente la existencia del banco de rescate fue el preludio y ejemplo para el futuro establecimiento de la Casa de Moneda. Pero no sólo eso, habrá que comparar, tanto a nivel anual como a nivel global, el monto de los canjes de rescate con el valor de la producción de plata zacatecana y con los ingresos de su caja real, conocer a los diversos personajes que cambiaron las platas, ubicarlos en el contexto social de la población, saber la importancia de sus canjes para poder evaluar el impacto real que tuvo la operación de los bancos de rescate.

\section{Barreaje.}

${ }^{38}$ ITESM, Campus Zacatecas, Zacatecas Collection, Treasury Records and Correspondance,

${ }^{39}$ Estos cuatro individuos cambiaron un total de 479 barras, que significó $69 \%$ del total del rescate. 
También sería importante saber, y esperamos que para ello se aboquen otros investigadores, cómo funcionaron los bancos de rescate en otros reales de minas. Saber las causas de su establecimiento, el periodo en que funcionaron, la importancia de sus operaciones, el impacto que pudo ello tener en la región, si operaron normalmente o en caso contrario las razones por las que dejaron de operar, son elementos que nos permitirán comprender de una mejor manera la conclusión del periodo borbónico y las causas de la revolución de independencia.

Las preguntas que realizamos en páginas anteriores se han respondido sólo parcialmente, por lo que es necesario continuar profundizando este estudio. 




